\title{
CARACTERÍSTICAS EDÁFICAS Y COMPOSICIÓN FLORÍSTICA DEL BOSQUE ESTACIONALMENTE SECO LA MENTA Y TIMBES, REGIÓN PIURA, PERÚ
}

\section{EDAPHYC CHARACTERISTICS AND FLORISTIC COMPOSITION OF THE SEASONALLY DRY FOREST LA MENTA AND TIMBES, PIURA REGION, PERU}

\author{
María Rasal Sánchez ${ }^{1}$, Joel Troncos Castro ${ }^{2}$, Carlos Lizano Durán ${ }^{3}$, Oscar Parihuamán Granda ${ }^{4}$, David Quevedo \\ Calle $^{5}$, Consuelo Rojas Idrogo ${ }^{6}$ y Guillermo E. Delgado Paredes ${ }^{7}$
}

\begin{abstract}
Resumen
Con el objetivo de contribuir al conocimiento de los ecosistemas naturales del país, se determinó las características edáficas y la composición florística del bosque estacionalmente seco (La Menta, $04^{\circ} 43^{\prime} 55^{\prime \prime} \mathrm{LS}$ y $80^{\circ} 04^{\prime} 55^{\prime \prime} \mathrm{LO}$, provincia de Piura y Timbes, $04^{\circ} 44^{\prime} 58^{\prime \prime}$ LS y $80^{\circ}$ 03' 33" LO, provincia de Ayabaca), ambos en la región Piura, Perú. El área de estudio abarcó una extensión aproximada de 6000 hectáreas en un rango altitudinal entre $350 \mathrm{~m}$ a $1350 \mathrm{~m}$. En cada bosque se muestrearon 5 transectos de 10 x $50 \mathrm{~m}$ a intervalos de $20 \mathrm{~m}$, sumando un total de 2500 $\mathrm{m}^{2}$. El análisis de suelo reveló niveles bajos de materia orgánica, nitrógeno y potasio y medios de fósforo asimilable. Estos suelos han sido clasificados en el orden Entisoles y suborden Orthents. En el bosque La Menta se registraron 18 especies, entre árboles y arbustos con diámetro a la altura del pecho $\geq 1.0 \mathrm{~cm}$, haciendo un total de 162 individuos, en tanto que en el bosque de Timbes se registraron 23 especies, sumando un total de 190 individuos. El área basal, por el área evaluada, fue de $128.86 \mathrm{~m}^{2}$ para La Menta, destacando Loxopterygium huasango con $45.97 \mathrm{~m}^{2}$ y $196.63 \mathrm{~m}^{2}$ para Timbes, destacando Erythrina smithiana con $47.13 \mathrm{~m}^{2}$. El Índice de Valor de Importancia, sobre un valor de 300.0 fue $50.0 \%$ para Bursera graveolens en La Menta y $62.0 \%$ para Cordia lutea en Timbes. El Índice de Diversidad de Shannon-Wiener mostró que el bosque La Menta fue de mayor valor que el bosque Timbes, aún cuando presentó menor número de especies, y la Matriz de Similaridad de Bray y Curtis indicó que ambos bosques tenían una similaridad de 54.68\%.

Palabras clave: Bosque estacionalmente seco, diversidad florística, características morfométricas,
\end{abstract} área basal, índice de similaridad.

\begin{abstract}
With the aim of contributing to the knowledge of the country's natural ecosistems, the edaphyc characteristics and the floristic composition of the seasonally dry forest, (La Menta, $04^{\circ}$ $43^{\prime} 55^{\prime \prime} \mathrm{LS}$ and $80^{\circ} 04^{\prime} 55^{\prime \prime} \mathrm{LO}$, province of Piura; and Timbes, $04^{\circ} 44^{\prime} 58^{\prime \prime} \mathrm{LS}$ and $80^{\circ} 03^{\prime} 33^{\prime \prime} \mathrm{LO}$, province of Ayabaca), both in the Piura Region, Peru, were determined. The study area covers an approximate area of 6000 hectares and has an altitude of 350 to 1350 m.a.s.l. In each forest 5 transects of $10 \times 50 \mathrm{~m}$ at intervals of $20 \mathrm{~m}$, were sampled, making a total of $2500 \mathrm{~m}^{2}$. Soil analysis showed low levels in organic matter, nitrogen and potassium and assimilable phosphorus media. These soils have been classified in the Entisols order and Orthents suborder. At La Menta forest 18 species were registered, among trees and shrubs with at breast height diameter $\mathrm{s} \geq 1.0 \mathrm{~cm}$, totaling a number of 162 individuals, whilst at Timbes forest 23 species were registered, with a total of 190 individuals. The basal area times total assessed area was $128.86 \mathrm{~m}^{2}$ for La Menta, where Loxopterigyum huasango with $45.97 \mathrm{~m}^{2}$ was highlighted. For Timbes it was $196.63 \mathrm{~m}^{2}$, where Erythrina smithiana with $47.13 \mathrm{~m}^{2}$ was highlighted. The importance value index, above a value of 300.0 was $50.0 \%$ for Bursera graveolens at La Menta and $62.0 \%$ for Cordia lutea at Timbes. The Diversity Index of Shannon-Wiener showed that the La Menta forest was of greater value than the Timbes forest, even though it had the lowest numbers of species and the Matrix of Bray and Curtis Similarity showed that the two forests had a similarity of $54.68 \%$.

Key words: Seasonally dry forest, floristic diversity, morphometric characteristics, basal area, similarity index.
\end{abstract}

\section{Introducción.}

El bosque seco ecuatorial o bosque estacionalmente seco (BES) es un ecosistema muy frágil que se extiende desde la península de Santa Elena, en el sur del Ecuador, hasta el noroeste del Perú, comprendiendo la costa de las regiones Tumbes, 
Piura, Lambayeque y el norte de La Libertad así como el piso inferior del valle del Marañón; estas dos áreas se comunican a través del Paso de Porculla, una depresión de $2100 \mathrm{~m}$ de elevación, considerada la más baja de los Andes peruanos (Brack \& Mendiola, 2004). El área del bosque seco es considerado una zona de importancia biológica por ser un ecosistema singular, muy amenazado y poco conocido, con presencia de especies endémicas y un importante grado de diversidad local y regional en una superficie relativamente reducida; por esta razón ha sido recientemente incluido entre los "puntos calientes" o hotspots del mundo, para su estudio y conservación (Mittermeier et al., 2005).

Las características edáficas de los BES, en contraste con los ecosistemas húmedos poco estacionales, son influenciadas por perturbaciones ambientales severas como la incidencia estacional y la sequía. La combinación de factores edáficos, la historia previa del uso de la tierra, la influencia de la vegetación remanente y la impredecibilidad de la sequía y el fuego, se combinan para producir múltiples rutas sucesionales (Chazdon, 2003). Entre estos factores, las variaciones edáficas locales y sus efectos sobre la trayectoria sucesional han recibido la menor atención (Leiva et al., 2009), aunque varios estudios indican que el suelo influencia la estructura, la diversidad y la abundancia de muchas especies de árboles tropicales (Clark et al., 1999).

Un estudio realizado por Sagástegui et al., (1999) ha revelado que la región norte y nororiente del Perú presenta cerca del $26 \%$ de especies vegetales endémicas diseminadas en varias comunidades: los bosques secos, las sabanas, los bosques de galerías y el algarrobal, destacando las especies de algarrobo [Prosopis pallida (Humb \& Bonpl. ex Willd.) Kunth], palo santo [Bursera graveolens (Kunth) Triana \& Planch.], hualtaco (Loxopterygium huasango Spruce ex Engler), zapote (Capparis scabrida H.B.K.), sauce (Salix humboldtiana Willdenow), pájaro bobo (Tessaria integrifolia R. \& P.), gigantón [Neoraimondia arequipensis (Meyen) Backeberg], salvajina [Tillandsia usneoides (L.) L.], ceibo [Ceiba insignis (Kunth) P.E. Gibbs \& Semir], entre otras, aún cuando para esta región del Perú se reporta a $C$. trichistandra Bakh. en lugar de C. insignis (La TorreCuadros \& Linares-Palomino, 2008). Complementariamente, un estudio realizado en los BES de Ecuador y Perú, importantes biológicamente porque forman el corazón de la Región de endemismo Tumbesina, permitió elaborar una lista conteniendo 313 especies leñosas (arbustos y árboles), de las cuales 239 especies se encuentran en Ecuador (136 reportadas sólo para Ecuador) y 177 en Perú (74 sólo para Perú), identificándose 66 especies endémicas, de las cuales 17 fueron exclusivas para Ecuador, 19 exclusivas para Perú y 30 compartidas en ambos países; las especies endémicas fueron el $20 \%$ del total, remarcando la importancia de la región (AguirreMendoza et al., 2006). Estudios recientes realizados por Linares-Palomino et al., (2010) en el bosque estacionalmente seco del Pacífico Ecuatorial, que comprende el oeste del Ecuador y el noroeste del Perú, determinaron que las regiones altas, entre 1000 y 1 $100 \mathrm{~m}$ de altitud resultaron más ricas en número total de especies y número de especies endémicas respecto a las regiones bajas, menores de $500 \mathrm{~m}$ de altitud, que resultaron comparativamente más pobres; de un total de 54 familias con 180 géneros y 313 especies, 67 especies $(21 \%$ del total) resultaron endémicas. Asimismo, el bosque seco del norte del Perú también presentó niveles similares de diversidad y endemismo en especies de vertebrados como es el caso del venado gris (Odocoileus virginianus Zimmermann 1780), oso hormiguero mediano (Tamandua mexicana Saussure 1860), oso de anteojos (Tremarctos ornatus Cuvier 1825), vizcacha (Lagidium peruanum Meyen 1833), pava aliblanca (Penelope albipennis Taczanowski 1878), chiroca (Icterus graceannae Cassin 1867), iguana (Iguana iguana Linnaeus 1758), macanche (Bothrops barnetti Parker 1938), sapo gigante (Bufo marinus Linnaeus 1758), entre otros (Aguilar, 1994). En el caso de Ecuador, recientemente se reportó la presencia de 5 especies de anfibios y 7 especies de reptiles en El Chilco, en el BES de la provincia de Loja (Armijos \& Valarezo, 2010). Como ha sido ampliamente enfatizado, el grado de endemismo se cuenta entre los principales criterios para clasificar la megadiversidad de un país, es por ello y por otras razones, por la que el Perú ha sido clasificado entre los 17 países con mayor megadiversidad (Mittermeier et al., 1997; WRI, 1997).

Por otro lado, no obstante la importancia del ecosistema del bosque seco ecuatorial y aún cuando pueden considerarse todavía escasos los estudios realizados y publicados en nuestro país, en los últimos años el avance ha sido significativo, destacando los trabajos de Zevallos (1986) quién realizó la caracterización dendrológica de 30 especies forestales de Lambayeque y de Bridgewater et al., (2003) quienes realizaron un análisis florístico $\mathrm{y}$ fitogeográfico preliminar de la flora leñosa del bosque seco en el norte del Perú, así como la publicación de valiosos documentos del Centro de Datos para la Conservación, por la Universidad Nacional Agraria La Molina (Lima), como el referido al estado de conservación de la diversidad natural de la región noroeste del Perú (CDC, 1992). Otros estudios han sido realizados en las regiones de Piura y Tumbes, así tenemos el estudio de los patrones de distribución espacial de Eriotheca ruizii (Schumann) Robyns, Bursera graveolens, Caesalpinia glabrata Kunth y Cochlospermum vitifolium (Willd.) Spreng., cuatro especies de árboles característicos de los bosques secos del Parque Nacional de Amotape, en el noroeste peruano, cuyos patrones no fueron significativamente 
diferentes de un patrón completamente al azar en varios de los casos analizados (Linares-Palomino, 2005); el análisis y discusión de los patrones de estructura y diversidad de los bosques secos estacionales de la Reserva de Biosfera del Noroeste, Parque Nacional Cerros de Amotape y Zona Reservada de Tumbes (Leal-Pinedo \& LinaresPalomino, 2005) y la identificación, en base a datos de inventarios florísticos de la vegetación estacional de las llanuras costeras de Piura, de seis grupos de vegetación que coincidieron ampliamente con los tipos de vegetación descritos por Augusto Weberbauer en varias publicaciones desde 1922-1945, lo que no sucedió con las ocho categorías de bosque seco definidas por el Proyecto Algarrobo para el área de estudio (Proyecto Algarrobo, 1993) donde ninguna pudo ser reconocida adecuadamente mediante métodos numéricos (La Torre-Cuadros \& LinaresPalomino, 2008). Además, se cuenta con los resultados de ocho inventarios botánicos rápidos realizados en los relictos del bosque tropical estacionalmente seco (BTES) de los alrededores de Jaén, Cajamarca, reportando 151 especies, 103 géneros y 40 familias de plantas leñosas y la existencia de un mayor número de endemismos respecto a otros BTES del Sur Occidente del Ecuador y Perú (Marcelo-Peña, 2008). En la Reserva Ecológica Chaparrí, Lambayeque, se inventarió todas las plantas con DAP $\geq 1.0 \mathrm{~cm}$ en una hectárea de bosque, registrándose 1255 individuos que representaron a 24 especies, 19 géneros y 12 familias, lo que permitió concluir que Chaparrí constituye una versión empobrecida de los BES en la región (LinaresPalomino \& Ponce-Álvarez, 2009); asimismo, se estudió la fenología de algunas especies que son alimento para la pava aliblanca Penelope albipennis (Martos et al., 2009). Adicionalmente, se cuenta con el trabajo de Linares-Palomino \& Pennington (2007) quienes elaboraron una lista anotada de plantas leñosas en bosques estacionalmente secos del Perú, constituyéndose en una valiosa herramienta en internet para estudios taxonómicos, ecológicos y de biodiversidad que ha permitido superar las deficiencias de escasa información biológica y nomenclatura ambigua, lo que con certeza limita elevar el perfil científico y de conservación de este tipo de bosques. En otros casos, los estudios se publicaron en informes internos de circulación restringida como lo son el inventario del potencial forestal del bosque seco Piedra del Toro, Morropón (PEBANINSA, 2003) y el del bosque seco de la comunidad campesina La Menta, Piura (HPI-PERÚ, 2007), ambos en la región Piura.

En el Ecuador, uno de los primeros trabajos se realizó en el Parque Nacional de Machalilla, un bosque seco semideciduo ubicado en el oeste y donde fueron reportadas 22 familias y 37 especies, destacando el género Ampeloceru sp. (Josse \&
Balslev, 1994); asimismo, se conocen varios trabajos realizados sobre la evaluación ecológica rápida de la vegetación en los bosques secos de la Ceiba $y$ Cordillera Arañitas, en la provincia de Loja (Aguirre et al., 2001), la vegetación de los bosques secos de Cerro Negro-Cazaderos, Occidente de la Provincia de Loja (Aguirre \& Delgado, 2005) y la composición florística y el estado de conservación de los bosques secos del sur-occidente del ecuador (Aguirre \& Kvist, 2005), así como el trabajo de Cerón et al., (2006) quienes realizaron un estudio sobre las características botánicas de la Reserva Militar y Ecológica Arenillas, El Oro, aunque esta formación vegetal incluye vegetación de manglares, espinares y sabanas.

El presente trabajo tiene como objetivo contribuir al conocimiento de los ecosistemas naturales del país, determinando las características edáficas y la composición florística de los bosques estacionalmente secos La Menta, en la provincia de Piura, y Timbes, en la provincia de Ayabaca, ambos en la región Piura, lo que con certeza facilitará la elaboración de planes de reforestación, conservación y utilización.

\section{Materiales y métodos.}

Las evaluaciones en el área de estudio se realizaron en el mes de abril del 2008.

Área de estudio: Fisiografía, hidrología y altitud.

El área de estudio se localizó entre las coordenadas, $04^{\circ} 43^{\prime} 55^{\prime \prime}$ LS a $04^{\circ} 44^{\prime} 58^{\prime \prime}$ LS y $80^{\circ} 03^{\prime} 33^{\prime \prime}$ LO a $80^{\circ}$ 04' 55" LO y correspondió al cerro El Fraile, una formación montañosa que comprende al BES La Menta, en el distrito de Las Lomas, provincia y región de Piura y al BES Timbes, en el distrito de Sapillica, provincia de Ayabaca y región Piura, extendiéndose en la parte alta y orilla izquierda del río Chipillico del valle San Lorenzo (Figura 1). Ambos bosques están adjudicados a comunidades campesinas del mismo nombre y comprenden 3919.41 ha para La Menta (HPI-PERU, 2007) y alrededor de 2000 ha para Timbes. El acceso al área de estudio fue por la carretera Piura-Sullana-Las Lomas, por la parte alta del reservorio de San Lorenzo, para luego ingresar a La Menta y Timbes, distante entre 30 a $50 \mathrm{~km}$ de Las Lomas. El área corresponde a estribaciones y contrafuertes de la cadena occidental montañosa de los Andes septentrionales, con colinas desde bajas hasta muy altas y quebradas donde discurren agua y sólidos en la época de lluvias intensas. La altitud va de 350 hasta $1350 \mathrm{~m}$ (cerro El Fraile).

Zonas de muestreo y vegetación.

El presente trabajo se realizó en un área ecológica que corresponde a la ecorregión del bosque seco ecuatorial (Brack \& Mendiola, 2004) y de acuerdo a la clasificación de zonas de vida de Holdridge (Holdridge, 1982) y al Mapa Ecológico del Perú (ONERN, 1976; INRENA-DGEPRN, 1998), corresponde al monte espinoso Tropical - mte-T, en las partes intermedias y monte espinoso premontano 
Tropical - mte-pT, en las partes altas; asimismo, se consideran dos comunidades vegetales, bosque ralo semicaducifolio y bosque semidenso caducifolio (CDC, 1992). Esta área se caracterizó por presentar vegetación mayoritariamente caducifolia, por citar el caso del palo santo (Bursera graveolens), porotillo (Erythrina smithiana) y hualtaco (Loxopterygium huasango), durante el largo periodo de sequía (mayodiciembre), como una estrategia de sobrevivencia, con excepción de algunas quebradas en las partes altas del cerro El Fraile donde se encuentran individuos perennifolios o siempreverdes. Asimismo, existe una profusa vegetación herbácea y arbustiva muy variada, que fue colectada entre los meses de febrero a mayo, así como algunas especies arbóreas no registradas en los transectos muestreados, mencionándose algunas de las más representativas: Alternanthera porrigens (Jacquin) Kuntze (moradilla) y A. brasiliana (L.) Kuntze (Amaranthaceae), Prestonia cordifolia Woodson (mataperro, platanitos), (Apocynaceae), Siegesbeckia flosculosa L'Héritier, Verbesina saubinetioides S.F. Blake y Pectis cajamarcana Keil (escobita) (Asteraceae), Delostoma integrifolium D. Don (babilla) (Bignoniaceae), Heliotropium angiospermum Murray (cola de alacrán) (Boraginaceae), Tillandsia usneoides (L.) L. (salvajina) (Bromeliaceae), Armatocereus cartwrightianus (Britton \& Rose) Backeberg ex A.W. Hill (cardo), Neoraimondia arequipensis (Meyen) Backeberg (gigantón) y Opuntia quitensis A. Weberbauer (tuna) (Cactaceae), Carica parviflora (A. DC.) Solms-Laubach (papaya silvestre) (Caricaceae), Ipomoea carnea Jacquin (borrachera) (Convolvulaceae), Euphorbia heterophylla L. (lecherita) (Euphorbiacaeae), Mimosa albida Humboldt \& Bonpland ex Willdenow (serría, tapatapa, uña de gato) (Fabaceae-Mimosoideae), Salvia occidentalis Swartz (Lamiaceae), Psittacanthus linearis (Killip) J.F. Macbride (suelda con suelda) (Loranthaceae), Sida weberbaueri Ulbrich (Malvaceae-Malvoideae), Mirabilis prostrata (R. \& P.) Heimerl (pega pega) (Nyctaginaceae), Oxalis dombeii A. St. Hilaire (chulco) (Oxalidaceae), Plumbago scandens L. (pega pega) (Plumbaginaceae), Panicum hirticaule J.S. Presl (chir chir) (Poaceae), Monnina sp. (Polygalaceae), Callisea repens L. (Scrophulariaceae), Acnistus arborescens (L.) Schlechtendal (chin chin, tuple), Grabowskia boerhaaviaefolia (L. f.) Schlechtendal (palo negro, canutillo) y Browalia americana L. (Solanaceae), Byttneria cordata Lamarck (MalvaceaeSterculioideae) y Lippia americana L. (Verbenaceae). Clima.

La información climatológica de temperatura fue proporcionada por la Estación Climatológica Ordinaria (CO) Partidor (04³8'38'S, 80¹5'15"W y $260 \mathrm{~m}$ de altitud), ubicada en el distrito de Las Lomas, provincia de Piura y región Piura y la de precipitación fue proporcionada por la Estación Pluviométrica (PLU) Sapillica (0447'00"S, 7959'00"W y 1540 m de altitud), ubicada en el distrito de Sapillica, provincia de Ayabaca y región Piura, encontrándose ambas estaciones en el ámbito geográfico de los BES La Menta y Timbes. La temperatura media anual (enero a diciembre/2008) fue $23.4{ }^{\circ} \mathrm{C}$, variando entre $21.4{ }^{\circ} \mathrm{C}$, media de junio y $25.5{ }^{\circ} \mathrm{C}$, media de marzo; la precipitación media anual fue $71.7 \mathrm{~mm}$, oscilando entre $0.0 \mathrm{~mm}$ en agosto y setiembre y 215.4 y 329.9 $\mathrm{mm}$ en marzo y abril, respectivamente.

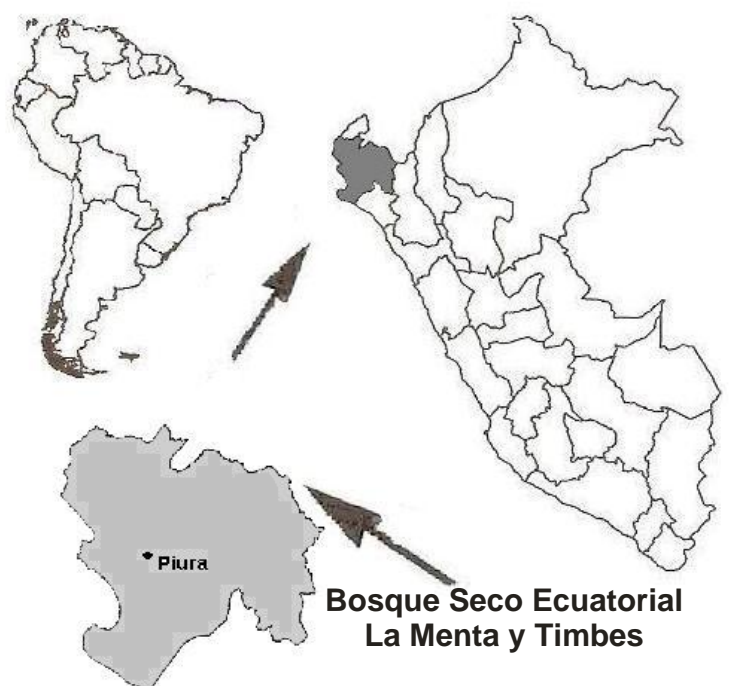

Figura 1. Ubicación del área de estudio. Bosque estacionalmente seco La Menta $\left(04^{\circ} 43^{\prime} 55^{\prime \prime}\right.$ LS y $80^{\circ} 04^{\prime} 55^{\prime \prime}$ LO) y Timbes $\left(04^{\circ} 44^{\prime} 58^{\prime \prime}\right.$ LS y $80^{\circ} 03^{\prime}$ 33" LO), Piura, Perú.

Muestreo.

El muestreo se realizó utilizando el método propuesto por Gentry (1982), con algunas modificaciones. En ambos bosques, La Menta y Timbes, se escogió un área con características fisiográficas y de vegetación uniformes, propendiendo a la unidad de paisaje o hábitat. El área de muestreo en La Menta se situó en los $04^{\circ} 43^{\prime}$ 55" LS, 80 04' 55" LO y entre $561-810 \mathrm{~m}$ de altitud y en Timbes se situó en los $04^{\circ} 44^{\prime} 58^{\prime \prime} \mathrm{LS}, 80^{\circ} 03^{\prime} 33^{\prime \prime}$ LO y entre 571-820 $\mathrm{m}$ de altitud. En cada área se delimitaron 5 transectos en orientación hacia la cima de la montaña, cada uno de 10 × $50 \mathrm{~m}$, a intervalos de $20 \mathrm{~m}$, haciendo un total de $2500 \mathrm{~m}^{2}$ de área de muestreo, es decir, $1 / 4 \mathrm{de}$ hectárea. Fueron muestreados árboles y arbustos con diámetro a la altura del pecho (DAP) $\geq 1.0 \mathrm{~cm}$. Asimismo, se midió la altura total, la altura comercial y el diámetro de copa. Todos los especímenes, fértiles y estériles, fueron colectados y herborizados para su correcta identificación en el laboratorio. Las muestras botánicas se depositaron en el Herbario de la Asociación Chira, Mallares (Sullana, Piura) (HACh), de reciente creación. En la determinación de la densidad (D) se consideró el número de individuos $\mathrm{x}$ 
Tabla 1. Análisis e interpretación del suelo realizados en cinco transectos del BES La Menta y Timbes, respectivamente.

\begin{tabular}{|c|c|c|}
\hline \multirow[t]{2}{*}{ Determinaciones* } & \multicolumn{2}{|c|}{ Resultados/Interpretación } \\
\hline & La Menta & Timbes \\
\hline Profundidad $(\mathrm{cm})$ & 30 & 30 \\
\hline Conductividad eléctrica (dS/m) & $0.50-0.72 /$ Muy ligera & $0.70-0.97 /$ Muy ligera \\
\hline pH (suelo/agua; 1:2,5) & $6.86-7.10 /$ Neutra & $7.13-7.45 /$ Neutra \\
\hline Calcáreo $\left(\mathrm{CaCO}_{3}\right)(\%)$ & $0.00-0.14 /$ Cero-Bajo & $0.22-0.52 /$ Bajo \\
\hline Materia orgánica (\%) & $0.45-0.68 /$ Bajo & $0.30-0.68 /$ Bajo \\
\hline Nitrógeno total $(\% \mathrm{~N})$ & $0.02-0.03 /$ Bajo & $0.01-0.03 /$ Bajo \\
\hline Fósforo asimilable (ppm P) & $10-11 /$ Medio & $9-10 /$ Medio \\
\hline Potasio (ppm K) & $141-150 /$ Bajo & $136-142 /$ Bajo \\
\hline Clase textural: $(\%)$ & Franco arcilloso/Fino & $\begin{array}{c}\text { Franco }- \text { Fr. } \\
\text { Arcilloso/Medio }- \text { Fino }\end{array}$ \\
\hline Arena & $33-41$ & $35-43$ \\
\hline Limo & $30-37$ & $28-38$ \\
\hline Arcilla & $29-34$ & $27-30$ \\
\hline C.I.C. $\mathrm{meq} / 100 \mathrm{~g}$ de & $13.92-15.59$ & $9.48-11.28$ \\
\hline $\mathrm{Ca}^{++} \mathrm{meq} / 100 \mathrm{~g}$ de suelo & $9.70-10.28$ & $6.48-8.08$ \\
\hline $\mathrm{Mg}^{++}$meq/100 g de suelo & $3.80-5.38$ & $2.38-2.78$ \\
\hline $\mathrm{K}^{+}$meq/100 g de suelo & $0.24-0.27$ & $0.27-0.32$ \\
\hline $\mathrm{Na}^{+}$meq/100 g de suelo & $0.12-0.20$ & $0.12-0.20$ \\
\hline $\mathrm{Da}\left(\mathrm{g} / \mathrm{cm}^{3}\right)$ & $1.32-1.36$ & $1.35-1.36$ \\
\hline \multicolumn{3}{|c|}{ N-P-K asimilable en $\mathrm{kg} / \mathrm{há} / \mathrm{año}$, en $30 \mathrm{~cm}$ de profundidad } \\
\hline $\mathrm{N}_{2}$ & $24-36$ & $12-37$ \\
\hline $\mathrm{P}_{2} \mathrm{O}_{5}$ & $91-102$ & $84-111$ \\
\hline $\mathrm{K}_{2} \mathrm{O}$ & $180-205$ & $327-385$ \\
\hline
\end{tabular}

* Determinado en el Laboratorio de Análisis de Suelo. Facultad de Agronomía, Universidad Nacional de Piura.

4, para expresarlo en hectárea, densidad relativa (Dr) densidad de una especie/densidad total, frecuencia (F) número de transectos donde se registra una especie/4, frecuencia relativa (Fr) frecuencia de una especie/frecuencia total, área basal $(\mathrm{AB})(\mathrm{DAP})^{2} \mathrm{x}$ 0.785, área basal relativa $(\mathrm{ABr}) \mathrm{AB}$ de una especie/AB total e Índice de Valor de Importancia (IVI\%) sumatoria de Dr, Fr y ABr y en todos los casos se utilizaron las fórmulas especificadas por Gentry (1982). En la medición de la diversidad alfa se utilizó el Índice de Riqueza Específica (IR) de Margalef; en la medición de la estructura fue utilizado el Índice de Dominancia (ID) de Simpson y, también, el Índice de Diversidad (H') de Shannon-Wiener (Magurran, 1988; Moreno, 2001). La diversidad beta se analizó a través de Matrices de Similaridad de Bray y Curtis (Bray \& Curtis, 1957). El análisis del suelo se realizó en el Laboratorio de Análisis de Suelo de la Facultad de Agronomía de la Universidad Nacional de Piura. En la determinación de la conductividad eléctrica, $\mathrm{pH}$, potasio y cationes cambiables se siguió las metodologías descritas por Jackson (1982) y en la determinación del fósforo, materia orgánica total, nitrógeno disponible, clase textural, carbonatos y la densidad aparente se siguió las metodologías descritas por López \& López (1990).

Tratamiento taxonómico.

El tratamiento taxonómico de las familias siguió los planteamientos filogenéticos del Grupo de Filogenia de las Angiospermas (APG, 1998; 2003). Así, se trata Fabaceae (incluyendo Caesalpinaceae, Mimosaceae y
Papilionaceae) y Malvaceae (incluyendo Bombacaceae, Sterculiaceae, Tiliaceae y Malvaceae) en sentido amplio.

\section{Resultados y discusión.}

Análisis del suelo.

El análisis del suelo mostró características muy similares entre el BES La Menta y el BES Timbes, con la significativa excepción en el $\mathrm{K}_{2} \mathrm{O}$ asimilable cuya concentración en Timbes duplicó a la determinada en La Menta; en ambos bosques la disponibilidad de materia orgánica y macronutrientes indispensables para las plantas, como nitrógeno, fósforo y potasio, se encontró en concentraciones de media a baja (Tabla 1), pero siempre siguiendo la misma tendencia decrescente: $\mathrm{Ca}, \mathrm{Mg}, \mathrm{K}$ y $\mathrm{Na}$, tal como fue observado en el contenido de estos elementos del suelo a lo largo de una cronosecuencia de regeneración del BES en Santa Rosa, Costa Rica, cuyos contenidos disminuyeron gradualmente en los bosques secundarios de 10, 15 y 20 años de edad para luego recuperarse hasta alcanzar niveles más altos en un bosque maduro mayor de 100 años (Leiva et al., 2009); la presencia de fósforo y potasio indicó la naturaleza mineralógica de los suelos analizados. En general, los suelos de los BES La Menta y Timbes presentaron poco desarrollo del horizonte superficial, con presencia de material no consolidado, ubicados en un clima seco y con vegetación propia de este ambiente ecológico, por lo tanto correspondió a la categoría Orden Entisoles, poco profundos $(<60 \mathrm{~cm})$ y por sus valores de arcilla y valores bajos de materia orgánica a la categoría del suborden Orthents, según el protocolo del Departamento de Agricultura de Estados Unidos (Soil Survey Staff, 2007) según el protocolo del Departamento de Agricultura de Estados Unidos (Soil Survey Staff, 2010) y lo reportado en el Atlas de Piura (Bernex de Falen \& Revesz, 1988). Dentro de los Entisoles poco profundos, Leiva et al., (2009) encontraron relaciones entre la edad del bosque y los niveles de $\mathrm{Ca}, \mathrm{Mg}, \mathrm{K}$ y $\mathrm{Na}$ intercambiables, lo que podría resultar en cambios en la diversidad de las especies a lo largo del proceso de regeneración, en vista de que especies con diferente fenología pueden preferir condiciones edáficas contrastantes (Goldberg, 1982). Los resultados obtenidos en los BES La Menta y Timbes indicaron la similaridad en la formación geológica de ambos suelos así como su exposición a los mismos procesos de erosión y degradación. 
En sitios sucesionales jóvenes, las quemas anuales de la vegetación herbácea pueden ser la causa de los aumentos generalizados en los contenidos de nutrimentos del suelo, principalmente de $\mathrm{Ca}, \mathrm{Mg}$ y $\mathrm{K}$ (Certini, 2005), tan igual como los pulsos de descomposición de hojarasca y raíces finas que adicionan materia orgánica $\mathrm{y}$ minerales al suelo (Cuevas, 1995), la fijación biológica de nitrógeno realizada por las Fabaceae (Gentry, 1995), como es el caso de la Erythrina smithina, abundante en el BES Timbes, entre otros factores; sin embargo, se ha indicado que la erosión eólica e hídrica causan la pérdida y lixiviación de bases, y la absorción de nutrimentos, por parte de las especies pioneras, reducen los niveles de bases del suelo (Leiva et al., 2009).

En un estudio reciente realizado en el BES Santa Rosa, Guanacaste (Costa Rica), se determinó que los suelos más comunes fueron los Entisoles (34\%) y los Vertisoles (21\%), aún cuando fueron identificados otros ordenes como Mollisoles, Alfisoles, Inceptisoles y Ultisoles (Leiva et al., 2009), y tal como ha sido reportado en nuestro estudio, presentaron bajo contenido de ceniza volcánica. Como ha sido ampliamente documentado, los efectos de la vegetación sobre el suelo son variados e influyen en los cambios de $\mathrm{pH}$, capacidad de intercambio catiónico y bases intercambiables, cantidad y calidad de la materia orgánica y contenido de nitrógeno (Russell et al., 2007; Leiva et al., 2009); complementariamente, conforme aumenta la edad de abandono del sitio, las especies arbóreas pioneras sustituyen el componente herbáceo, aumenta la adición anual de hojarasca y raíces finas, se conserva mejor la humedad y se enriquece la superficie del suelo con cationes y materia orgánica (Alfaro et al., 2001). Es posible que esta dinámica estaría ocurriendo en los BES La Menta y Timbes.

Identificación de especies.

En la época en que se realizó el muestreo se encontró muy pocas muestras fértiles, por lo tanto, resultó difícil la identificación hasta nivel específico para un número significativo de éstas, razón por la cual fue necesario retornar al bosque varias veces a lo largo del año para colectar muestras fértiles, en los especímenes previamente marcados, y hacer las comparaciones correspondientes. En tal sentido resultó válido lo señalado por Wilkie et al., (2004) cuando afirmaron que los datos florísticos precisos de parcelas completas son por lo general obtenidos después de varios años de colecta continua; y con certeza, la recolección continua, sobre todo de las especies no identificadas o en duda, incrementarán el conocimiento sobre la flora leñosa del lugar (LealPinedo \& Linares-Palomino, 2005).

Composición florística.

Como se observa en la tabla 2, en los 5 transectos muestreados en el BES La Menta fueron determinados
162 individuos (648/ha), entre árboles y arbustos, con un DAP $\geq 1.0 \mathrm{~cm}$, correspondiendo a 18 especies, 16 géneros y 15 familias. El mayor número de individuos correspondió a Cordia lutea con 38, seguido de Bursera graveolens con 22, Tecoma stans con 20, Coccoloba ruiziana con 15 y Eriotheca ruizii con 14 individuos. El mayor DAP correspondió a Loxopterygium huasango con $41.2 \mathrm{~cm}$ seguido de $B$. graveolens y Caesalpinia glabrata con $29.6 \mathrm{~cm}$, por especie, aunque $L$. huasango apenas registró 3 individuos. Los árboles de mayor tamaño fueron, también, L. huasango con $10.0 \mathrm{~m}$ de altura seguido de B. graveolens y E. ruizii con 8.3 y $8.1 \mathrm{~m}$, respectivamente, correspondiéndoles, asimismo, el mayor diámetro de copa con 8.9; 6.5 y $6.7 \mathrm{~m}$, respectivamente. Tres especies, C. lutea, $B$. graveolens y $C$. ruiziana, se observaron en los 5 transectos muestreados en tanto que 6 especies, Capparis avicennifolia, Capparis scabrida, C. glabrata, Geoffroea striata, Schrebera americana y Ximenia americana, se observaron en apenas un transecto muestreado.

$\mathrm{Si}$ bien es cierto que el mayor número de individuos correspondió a las especies arbustivas, Cordia lutea, Coccoloba ruiziana y Cordia macrocephala, con más de un tercio $(40.7 \%)$ del total de individuos muestreados, resultó significativa la presencia de árboles adultos de Bursera graveolens y Eriotheca ruizii con 13.6 y $8.6 \%$, en cada caso, y árboles muy jóvenes de Tecoma stans con $12.3 \%$, del total de individuos muestreados. Por otro lado, resultó sorprendente la mínima presencia de Loxopterygium huasango, Caesalpinia glabrata, Acacia macracantha, Geoffroea striata, Schrebera americana, Ximenia americana y Capparis scabrida, con apenas $1-3$ individuos muestreados y la ausencia total de Tabebuia billbergii (Guayacán), Ceiba trichistandra (Barrigón), Cochlospermum vitifolium (Polo polo), Pithecellobium excelsum (Chaquiro), Erythrina smithiana (Porotillo) y Celtis iguanaea (Jacq.) Sarg. (Palo blanco), importantes especies madereras del bosque seco.

Como se observa en la tabla 3 , en los 5 transectos muestreados en el BES Timbes fueron determinados 190 individuos (760/ha), entre árboles y arbustos, con un DAP $\geq 1.0 \mathrm{~cm}$, correspondiendo a 23 especies, 22 géneros y 19 familias. El mayor número de individuos correspondió a Cordia lutea con 95, seguido de Erythrina smithiana con 15, Bursera graveolens con 10 y Cestrum auriculatum y Cassia sp. con 8 individuos, respectivamente. El mayor DAP correspondió a Ceiba trichistandra con 70,6 cm seguido de Leucaena trichodes, Eriotheca ruizii y E. smithiana con $54.1 ; 42.8$ y $36.4 \mathrm{~cm}$, respectivamente, aunque $E$. ruizii y $L$. trichodes apenas registró 3 y 1 individuos. Los árboles de mayor tamaño fueron $C$. trichistandra con $14.1 \mathrm{~m}$ de altura seguido de $E$. smithiana, Loxopterygium huasango y 
Cochlospermum vitifolium con 10.4; 9.5 y $9.0 \mathrm{~m}$ respectivamente; sin embargo, el mayor diámetro de copa correspondió a $C$. trichistandra, B. graveolens y E. smithiana con $10.6 ; 7.8$ y $7.1 \mathrm{~m}$, respectivamente. Únicamente $C$. lutea se observó en los 5 transectos muestreados en tanto que entre las especies arbóreas 6 de éstas, C. trichistandra, Myrcianthes sp., $L$. trichodes, T. stans, C. vitifolium y Capparis scabrida, se observaron en apenas un transecto muestreado.

$\mathrm{Si}$ bien es cierto que el mayor número de individuos correspondió a la especie arbustiva Cordia lutea, con $50.6 \%$ del total de individuos muestreados, resultó significativa la presencia de árboles adultos de Erytrhina smithiana, Bursera graveolens y Ceiba trichistandra con $7.9 ; 5.3$ y $3.7 \%$, en cada caso, del total de individuos muestreados. Por otro lado, resultó muy pobre la presencia de Loxopterygium huasango,
Ximenia americana, Capparis scabrida, Eriotheca ruizii y Cochlospermum vitifolium, con apenas 1 3 individuos muestreados y la ausencia total de Tabebuia billbergii, Pithecellobium excelsum, Caesalpinia glabrata, Acacia macracantha, Geoffroea striata, Schrebera americana y Celtis iguanaea, especies madereras muy valiosas del bosque seco.

En relación a los resultados mostrados en las tablas 2 y 3 , éstos se ajustaron mejor con los resultados mostrados para el bosque semidenso de montaña (Bssm) del BES La Menta, en el reporte de HPI-PERÚ (2007), aún cuando la comparación tiene un sesgo significativo puesto que en dicho reporte se indicó la ocurrencia de 138 individuos/ha, pero de especies únicamente arbóreas, es decir, sin tomar en consideración las especies arbustivas, que por cierto tienen una significativa presencia en el bosque;

Tabla 2. Principales características fisonómicas y hábito de crecimiento de las especies muestreadas en el BES La Menta.

\begin{tabular}{|c|c|c|c|c|c|c|c|c|c|c|c|c|}
\hline \multirow[t]{2}{*}{$\mathbf{N}^{\mathbf{0}}$} & \multirow[t]{2}{*}{$\begin{array}{l}\text { Especie } \\
\text { Nombre vulgar/Familia }\end{array}$} & \multirow[t]{2}{*}{ Hb. } & \multirow[t]{2}{*}{$\mathbf{N}^{0}$ Indi. } & \multicolumn{5}{|c|}{ Muestreo } & \multicolumn{4}{|c|}{$\begin{array}{l}\text { Características } \\
\text { fisonómicas }\end{array}$} \\
\hline & & & & 1 & 2 & 3 & 4 & 5 & $\begin{array}{l}\text { DAP } \\
(\mathbf{c m})\end{array}$ & $\begin{array}{l}\text { HT } \\
(\mathbf{m})\end{array}$ & $\begin{array}{l}\text { HC } \\
(\mathbf{m})\end{array}$ & $\begin{array}{l}\text { DC } \\
(\mathbf{m})\end{array}$ \\
\hline 1 & $\begin{array}{l}\text { Acacia macracantha Humb. \& Bonpl. Ex Willd } \\
\text { Faique/Fabaceae }\end{array}$ & $\mathrm{Ab}$. & 2 & $\mathrm{x}$ & $\mathrm{x}$ & & & & 9.4 & 4.2 & 0.0 & 3.5 \\
\hline 2 & $\begin{array}{l}\text { Bougainvillea pachyphylla Heimerl ex Standl. } \\
\text { Papelillo/Nyctaginaceae }\end{array}$ & Ar. & 14 & $\mathrm{x}$ & $\mathrm{x}$ & & $\mathrm{x}$ & $\mathrm{x}$ & 2.6 & 2.9 & 0.0 & 2.2 \\
\hline 3 & $\begin{array}{l}\text { Bursera graveolens (Kunth) Triana \& Planch. } \\
\text { Palo santo/Burseraceae }\end{array}$ & $\mathrm{Ab}$. & 22 & $\mathrm{x}$ & $\mathrm{x}$ & $\mathrm{x}$ & $\mathrm{x}$ & $\mathrm{x}$ & 29.7 & 8.3 & 3.9 & 6.5 \\
\hline 4 & $\begin{array}{l}\text { Caesalpinia glabrata Kunth } \\
\text { Charán/Fabaceae }\end{array}$ & $\mathrm{Ab}$. & 2 & & & $\mathrm{x}$ & & & 29.6 & 7.5 & 2.8 & 7.2 \\
\hline 5 & $\begin{array}{l}\text { Capparis avicennifolia Kunth } \\
\text { Vichayo/Capparaceae }\end{array}$ & Ar. & 1 & & $\mathrm{x}$ & & & & 10.2 & 4.1 & 0.0 & 4.1 \\
\hline 6 & $\begin{array}{l}\text { Capparis scabrida Kunth } \\
\text { Zapote/Capparaceae }\end{array}$ & $\mathrm{Ab}$. & 1 & & & & $\mathrm{x}$ & & 6,1 & 3,5 & 0.0 & 3.0 \\
\hline 7 & $\begin{array}{l}\text { Cissus sp. } \\
\text { Bombacea/Vitaceae }\end{array}$ & & 5 & $\mathrm{x}$ & $\mathrm{x}$ & $\mathrm{x}$ & & & 4.85 & 0.0 & 0.0 & 0.0 \\
\hline 8 & $\begin{array}{l}\text { Coccoloba ruiziana Lindau } \\
\text { Añalque/Polygonaceae }\end{array}$ & Ar. & 15 & $\mathrm{x}$ & $\mathrm{x}$ & $\mathrm{x}$ & $\mathrm{x}$ & $\mathrm{x}$ & 14.4 & 4.8 & 0.0 & 5.9 \\
\hline 9 & $\begin{array}{l}\text { Cordia lutea Lam. } \\
\text { Overo/Boraginaceae }\end{array}$ & Ar. & 38 & $\mathrm{x}$ & $\mathrm{x}$ & $\mathrm{x}$ & $\mathrm{x}$ & $\mathrm{x}$ & 8.9 & 3.2 & 0.0 & 3.2 \\
\hline 10 & $\begin{array}{l}\text { Cordia macrocephala (Desv.) Kunth } \\
\text { Uta/Boraginaceae }\end{array}$ & Ar. & 13 & & $\mathrm{x}$ & $\mathrm{x}$ & $\mathrm{x}$ & & 4.1 & 3.0 & 0.0 & 3.4 \\
\hline 11 & $\begin{array}{l}\text { Croton collinus H.B.K. } \\
\text { Mosquera/Euphorbiaceae }\end{array}$ & Ar. & 4 & & $\mathrm{x}$ & $\mathrm{x}$ & $\mathrm{x}$ & $\mathrm{x}$ & 1.5 & 1.5 & 0.0 & 1.7 \\
\hline 12 & $\begin{array}{l}\text { Eriotheca ruizii (K. Schum.) A. Robyns } \\
\text { Pasayo/Malvaceae }\end{array}$ & $\mathrm{Ab}$. & 14 & & & $\mathrm{x}$ & $\mathrm{x}$ & $\mathrm{x}$ & 23.8 & 8.1 & 4.0 & 6.7 \\
\hline 13 & $\begin{array}{l}\text { Geoffroea striata (Willd.) Morong } \\
\text { Almendro/Fabaceae }\end{array}$ & $\mathrm{Ab}$. & 1 & & $\mathrm{x}$ & & & & 5.7 & 3.0 & 0.0 & 1.9 \\
\hline 14 & $\begin{array}{l}\text { Loxopterygium huasango Spruce ex Engl./ } \\
\text { Hualtaco/Anacardiaceae }\end{array}$ & $\mathrm{Ab}$. & 3 & & $\mathrm{x}$ & & $\mathrm{x}$ & $\mathrm{x}$ & 41.2 & 10.0 & 5.3 & 8.9 \\
\hline 15 & $\begin{array}{l}\text { Schrebera americana (Zahlbr.) Gilg } \\
\text { Diente de león/Oleaceae }\end{array}$ & $\mathrm{Ab}$. & 1 & & $\mathrm{x}$ & & & & 13.1 & 8.0 & 3.5 & 4.6 \\
\hline 16 & $\begin{array}{l}\text { Senna mollisima (Willd.) Irwin \& Barneby } \\
\text { Chaquiro liso/Fabaceae }\end{array}$ & $\mathrm{Ab}$. & 5 & $\mathrm{x}$ & $\mathrm{x}$ & $\mathrm{x}$ & $\mathrm{x}$ & & 8.6 & 6.8 & 0.5 & 3.8 \\
\hline 17 & $\begin{array}{l}\text { Tecoma stans (L.) Juss. Ex Kunth } \\
\text { Guayacancillo/Bignoniaceae }\end{array}$ & $\mathrm{Ab}$ & 20 & $\mathrm{x}$ & & $\mathrm{x}$ & $\mathrm{x}$ & $\mathrm{x}$ & 6.4 & 3.1 & 0.0 & 2.7 \\
\hline 18 & $\begin{array}{l}\text { Ximenia americana } \mathrm{L} \text {. } \\
\text { Limoncillo/Olacaceae }\end{array}$ & $\mathrm{Ab}$ & 1 & & $\mathrm{x}$ & & & & 23.9 & 7.0 & 0.0 & 4.6 \\
\hline
\end{tabular}

Fam., familia; Hb., hábito de crecimiento; $\mathrm{N}^{0}$ Indi., número de individuos; DAP, diámetro a la altura del pecho; HT, altura total; HC, altura comercial; DC, diámetro de la copa; Ab., árbol; Ar., arbusto. 
además, hay que tener en consideración que dicho estudio se realizó en 15 transectos de 20 x $500 \mathrm{~m}$ cada uno $(0.37 \%$ del área total del bosque) y partiendo con árboles con DAP $\geq 10 \mathrm{~cm}$, destacando $E$. ruizii (15.4\%), B. graveolens (11.3\%) y A. macracantha $(8.6 \%)$, alcanzando alrededor de $72 \%$ los árboles con $10-30 \mathrm{~cm}$ por clase diamétrica; sin embargo, existe coincidencia en asumir que el bosque ha sido severamente intervenido por la explotación irracional y selectiva de las especies arbóreas más valiosas.

Resultó significativa la presencia de un alto porcentaje de familias monotípicas representadas por una especie leñosa (33.3\% en el BES La Menta y $30.4 \%$ en el BES Timbes), lo cual indicó una alta tasa de diversidad beta y reconfirmó el alto grado de heterogeneidad de los bosques secos peruanos, tal

Tabla 3. Principales características fisonómicas y hábito de crecimiento de las especies muestreadas en el BES Timbes.

\begin{tabular}{|c|c|c|c|c|c|c|c|c|c|c|c|c|}
\hline \multirow[t]{2}{*}{$\mathbf{N}^{\mathbf{0}}$} & \multirow{2}{*}{$\begin{array}{l}\text { Especie } \\
\text { Nombre vulgar / Familia }\end{array}$} & \multirow[t]{2}{*}{ Hb. } & \multirow{2}{*}{$\begin{array}{c}\mathbf{N}^{\mathbf{0}} \\
\text { Indi. }\end{array}$} & \multicolumn{5}{|c|}{ Muestreo } & \multicolumn{4}{|c|}{ Características fisonómicas } \\
\hline & & & & 1 & 2 & 3 & 4 & 5 & $\begin{array}{l}\text { DAP } \\
\text { (cm) }\end{array}$ & $\begin{array}{l}\text { HT } \\
(\mathbf{m})\end{array}$ & $\begin{array}{l}\text { HC } \\
(\mathbf{m})\end{array}$ & $\begin{array}{l}\text { DC } \\
(\mathbf{m})\end{array}$ \\
\hline 1 & $\begin{array}{l}\text { Bougainvillea pachyphylla Heimerl ex Standl. } \\
\text { Papelillo / Nyctaginaceae }\end{array}$ & Ar. & 3 & & $\mathrm{x}$ & & $\mathrm{x}$ & & 3.9 & 4.5 & 0.0 & 2.3 \\
\hline 2 & $\begin{array}{l}\text { Bursera graveolens (Kunth) Triana \& Planch. } \\
\text { Palo santo / Burseraceae }\end{array}$ & $\mathrm{Ab}$ & 10 & $\mathrm{x}$ & $\mathrm{x}$ & $\mathrm{x}$ & $\mathrm{x}$ & & 21.6 & 8.3 & 0.0 & 7.8 \\
\hline 3 & $\begin{array}{l}\text { Capparis scabrida Kunth } \\
\text { Zapote / Capparaceae }\end{array}$ & $\mathrm{Ab}$. & 1 & & & & $\mathrm{x}$ & & 21.7 & 7.0 & 0.0 & 5.0 \\
\hline 4 & $\begin{array}{l}\text { Cassia sp. } \\
\text { Fabaceae }\end{array}$ & $\mathrm{Ab}$. & 8 & & $\mathrm{x}$ & $\mathrm{x}$ & $\mathrm{x}$ & & 3.4 & 4.1 & 0.0 & 1.8 \\
\hline 5 & $\begin{array}{l}\text { Ceiba trichistandra (A. Gray) Bakh. } \\
\text { Barrigón / Malvaceae }\end{array}$ & $\mathrm{Ab}$ & 7 & $\mathrm{x}$ & & & & & 70.6 & 14.1 & 0.0 & 10.6 \\
\hline 6 & $\begin{array}{l}\text { Cestrum auriculatum } \mathrm{L}^{\prime} \mathrm{Her} \\
\text { Hierba santa / Solanaceae }\end{array}$ & Ar. & 8 & $\mathrm{x}$ & $\mathrm{x}$ & $\mathrm{x}$ & & & 2.8 & 3.5 & 0.0 & 2.5 \\
\hline 7 & $\begin{array}{l}\text { Cissus sp. } \\
\text { Bombacea / Vitaceae }\end{array}$ & & 7 & $\mathrm{x}$ & $\mathrm{x}$ & $\mathrm{x}$ & $\mathrm{x}$ & & 8.1 & 12.8 & 0.0 & 0.0 \\
\hline 8 & $\begin{array}{l}\text { Coccoloba ruiziana (Lindau) } \\
\text { Añalque / Polygonaceae }\end{array}$ & Ar. & 2 & & & $\mathrm{x}$ & $\mathrm{x}$ & & 12.5 & 5.0 & 0.0 & 4.5 \\
\hline 9 & $\begin{array}{l}\text { Cochlospermum vitifolium (Willd.) Spreng. } \\
\text { Polo polo / Cochlospermaceae }\end{array}$ & $\mathrm{Ab}$ & 1 & $\mathrm{x}$ & & & & & 13.7 & 9.0 & 0.0 & 3.5 \\
\hline 10 & $\begin{array}{l}\text { Cordia lutea Lam. } \\
\text { Overo / Boraginaceae }\end{array}$ & Ar. & 95 & $\mathrm{x}$ & $\mathrm{x}$ & $\mathrm{x}$ & $\mathrm{x}$ & $\mathrm{x}$ & 10.9 & 3.5 & 0.0 & 3.2 \\
\hline 11 & $\begin{array}{l}\text { Cordia macrocephala (Desv.) Kunth } \\
\text { Uta / Boraginaceae }\end{array}$ & Ar. & 3 & $\mathrm{x}$ & $\mathrm{x}$ & & & & 3.7 & 2.9 & 0.0 & 1.9 \\
\hline 12 & $\begin{array}{l}\text { Croton collins H.B.K. } \\
\text { Mosquera / Euphorbiaceae }\end{array}$ & Ar. & 5 & & $\mathrm{x}$ & $\mathrm{x}$ & $\mathrm{x}$ & & 1.3 & 3.6 & 0.0 & 1.8 \\
\hline 13 & $\begin{array}{l}\text { Eriotheca ruizii (K. Schum.) A. Robyns } \\
\text { Pasayo / Malvaceae }\end{array}$ & $\mathrm{Ab}$ & 3 & & & $\mathrm{x}$ & $\mathrm{x}$ & & 42.8 & 8.5 & 4.6 & 7.0 \\
\hline 14 & $\begin{array}{l}\text { Erythrina smithiana Krukoff } \\
\text { Porotillo / Fabaceae }\end{array}$ & $\mathrm{Ab}$ & 15 & $\mathrm{x}$ & $\mathrm{x}$ & $\mathrm{x}$ & $\mathrm{x}$ & & 36.4 & 10.4 & 4.6 & 7.1 \\
\hline 15 & $\begin{array}{l}\text { Leucaena trychodes (Jacq.) Benth. } \\
\text { Chapra / Fabaceae }\end{array}$ & $\mathrm{Ab}$ & 1 & $\mathrm{x}$ & & & & & 54.1 & 7.0 & 5.0 & 5.3 \\
\hline 16 & $\begin{array}{l}\text { Loxopterygium huasango (Spruce ex Engl. } \\
\text { Hualtaco / Anacardiaceae }\end{array}$ & $\mathrm{Ab}$ & 2 & $\mathrm{x}$ & & $\mathrm{x}$ & & & 27.7 & 9.5 & 4.5 & 5.0 \\
\hline 17 & $\begin{array}{l}\text { Myrcianthes sp. } \\
\text { Myrtaceae }\end{array}$ & $\mathrm{Ab}$ & 3 & & & & $\mathrm{x}$ & & 2.3 & 3.5 & 0.0 & 1.3 \\
\hline 18 & $\begin{array}{l}\text { Pisonia macranthocarpa J.D. Smith } \\
\text { Pego pego / Nyctaginaceae }\end{array}$ & Ar. & 4 & $\mathrm{x}$ & $\mathrm{x}$ & $\mathrm{x}$ & $\mathrm{x}$ & & 2.8 & 3.3 & 0.0 & 2.2 \\
\hline 19 & $\begin{array}{l}\text { Senna mollisima (Willd.) Irwin \& Barneby } \\
\text { Chaquiro liso / Fabaceae }\end{array}$ & $\mathrm{Ab}$ & 7 & $\mathrm{x}$ & & $\mathrm{x}$ & $\mathrm{x}$ & & 8.6 & 6.8 & 0.5 & 3.8 \\
\hline 20 & $\begin{array}{l}\text { Sida paniculata (L.) Fryxell } \\
\text { Pichana / Malvaceae }\end{array}$ & Ar. & 1 & $\mathrm{x}$ & & & & & 1.3 & 2.0 & 0.0 & 1.4 \\
\hline 21 & $\begin{array}{l}\text { Solanum amotapense Svenson } \\
\text { Tomatillo / Solanaceae }\end{array}$ & $\mathrm{Ab}$ & 1 & $\mathrm{x}$ & & & & & 2.9 & 3.5 & 0.0 & 2.0 \\
\hline 22 & $\begin{array}{l}\text { Tecoma stans (L.) Juss. Ex Kunth } \\
\text { Guayacancillo / Bignoniaceae }\end{array}$ & $\mathrm{Ab}$ & 1 & $\mathrm{x}$ & & & & & 16.2 & 6.0 & 0.0 & 4.0 \\
\hline 23 & $\begin{array}{l}\text { Ximenia americana L. } \\
\text { Limoncillo / Olacaceae }\end{array}$ & $\mathrm{Ab}$ & 2 & $\mathrm{x}$ & & & $\mathrm{x}$ & & 15.8 & 6.0 & 0.0 & 4.1 \\
\hline
\end{tabular}

Fam., familia; Hb., hábito de crecimiento; $\mathrm{N}^{0}$ Indi., número de individuos; DAP, diámetro a la altura del pecho; HT, altura total; HC, altura comercial; DC, diámetro de la copa; Ab., árbol; Ar., arbusto. 
como ya había sido observado por Bridgewater et al., (2003) al comparar bosques secos del norte peruano, incluyendo los del valle del Marañón y de los alrededores de Tarapoto y por Leal-Pinedo \& LinaresPalomino et al., (2005), quienes estudiando los bosques secos de la Reserva de Biosfera del Noroeste del Perú determinaron $32 \%$ de familias monotípicas, un valor muy similar a los reportados en nuestro trabajo. Complementariamente, en el estudio realizado en el BES Reserva Ecológica Chaparrí (Lambayeque), el inventario de todas las plantas con un DAP $\geq 1.0$ $\mathrm{cm}$ en una hectárea de bosque se registró 1255 individuos que representaron a 24 especies, 19

géneros y 12 familias; la especie más abundante fue Cordia lutea (Boraginaceae, 249 individuos), seguido por Ditaxis dioica (Euphorbiaceae, 177 individuos), Eriotheca ruizii (MalvaceaeBombacoideae, 151 individuos), Bursera graveolens (Burseraceae, 125 individuos) y Praecereus euchlorus (Cactaceae, 100 individuos), contribuyendo con 64\% del número total de individuos y $58 \%$ del área basal total; el género más diverso fue Capparis (Capparaceae) y la familia más diversa fue Leguminosae (Fabaceae) (Linares-Palomino \& PonceÁlvarez, 2009); esta composición florística, con excepción de $D$. dioica y $P$. euchlorus, se asemejó significativamente con los resultados de nuestro trabajo, en especial por la presencia de numerosos individuos de C. lutea.

Clase diamétrica, área basal e índice de valor de importancia.

En referencia a la clase diamétrica, en el BES La Menta, de un total de 162 individuos muestreados, 88 $(54.3 \%)$ correspondió al rango $0-10 \mathrm{~cm}$, destacando sólo dos individuos, uno de Loxopterygium huasango y otro de Bursera graveolens, en el rango de 50-60 cm de clase diamétrica. En el BES Timbes, de un total de 190 individuos muestreados, $106 \quad$ (55.8\%) correspondió al rango $0-10 \mathrm{~cm}$, destacando únicamente tres individuos, dos de Ceiba trcihistandra y uno de Eriotheca ruizii, en el rango de $80-90 \mathrm{~cm}$ de clase diamétrica (Tabla 4).

Estos resultados coincidieron en parte con los reportados por HPI-PERÚ (2007) sobre el bosque seco semidenso de montaña (Bss-m) y el bosque seco denso de montaña (Bsd-m), ambos en La Menta, puesto que en la clase diamétrica 10 $20 \mathrm{~cm}$ se registró $43.9 \%$ de un total de 138 árboles/ha, para el primer caso, y $37.6 \%$ de un total de 160 árboles/ha, para el segundo correspondió
Tabla 4. Número de árboles muestreados por clase diamétrica de las especies con los valores más altos en los BES La Menta y Timbes.

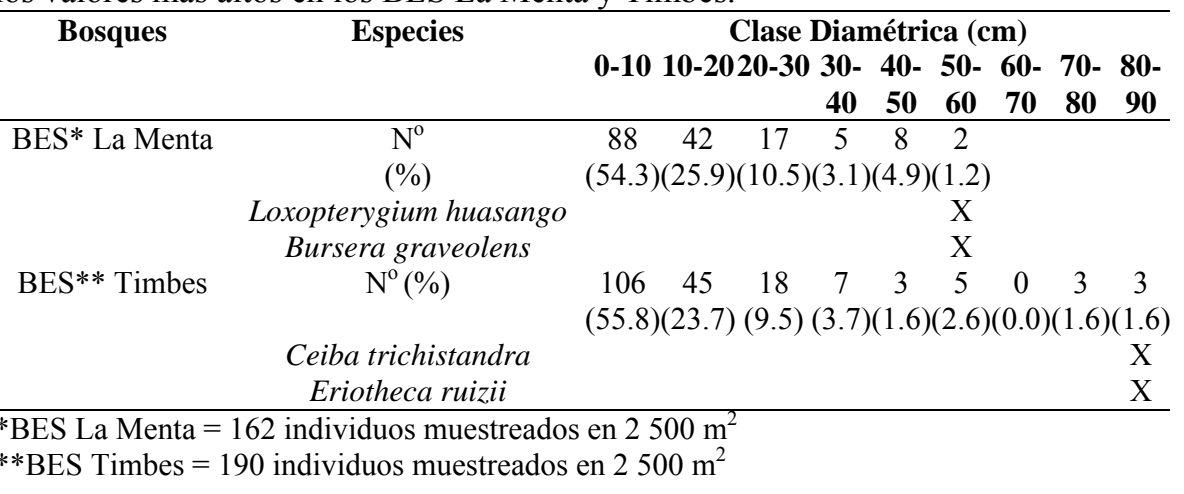


dioica, y estas Tabla 5. Área basal (AB) e Índice de valor de importancia (IVI) de las especies muestreadas cinco especies en el BES La Menta.

\begin{tabular}{|c|c|c|c|c|c|c|c|c|c|c|c|}
\hline \multirow{4}{*}{$\begin{array}{l}\text { contenían } 59 \% \\
\text { del número de } \\
\text { individuos y más } \\
\text { de } 90 \% \text { del área } \\
\text { basal } \\
\text { (Linares- }\end{array}$} & $\mathbf{N}^{\mathbf{0}}$ & Especie & $\begin{array}{c}\mathbf{N}^{\mathbf{0}} \\
\text { Indiv. }\end{array}$ & $\begin{array}{l}\mathbf{N}^{\mathbf{0}} \\
\mathbf{I} / \mathbf{T}\end{array}$ & D & Dr & $\mathbf{F}$ & $\mathbf{F r}$ & $\begin{array}{c}\text { AB } \\
\left(\mathbf{m}^{2}\right)\end{array}$ & $\mathrm{ABr}$ & $\begin{array}{l}\text { IVI } \\
(\%)\end{array}$ \\
\hline & $\overline{1 A}$ & Acacia macracantha & 2 & 2 & 8 & 0.01 & 0.50 & 0.04 & 2.18 & 0.002 & 7.0 \\
\hline & & Bougainvillea pachyphylla & 14 & 4 & 56 & 0.09 & 1.00 & 0.08 & 0.24 & 0.002 & 17.0 \\
\hline & & Bursera graveolens & 22 & 5 & 88 & 0.14 & 1.25 & 0.10 & 34.69 & 0.269 & 50.0 \\
\hline Palomino \& & $4 c$ & Caesalpinia glabrata & 2 & 1 & 8 & 0.01 & 0.25 & 0.02 & 6.83 & 0.053 & 8.0 \\
\hline Ponce-Álvarez, & & Capparis avicennifolia & 1 & 1 & 4 & 0.01 & 0.25 & 0.02 & 0.81 & 0.006 & 3.0 \\
\hline Estos & $6 c$ & Capparis scabrida & 1 & 1 & 4 & 0.01 & 0.25 & 0.02 & 0.29 & 0.002 & 3.0 \\
\hline resultados fueron & 7 & Cissus sp. & 5 & 3 & 20 & 0.03 & 0.75 & 0.06 & 0.57 & 0.004 & 9.0 \\
\hline similares a los & 8 & Coccoloba ruiziana & 15 & 5 & 60 & 0.09 & 1.25 & 0.09 & 8.47 & 0.066 & 26.0 \\
\hline nuestros en lo que & 9 & Cordia lutea & 38 & 5 & 152 & 0.24 & 1.25 & 0.09 & 3.38 & 0.026 & 36.0 \\
\hline spect & & Cordia macrocep & 13 & 3 & 52 & 0.08 & 0.75 & 0.06 & 0.50 & 0.004 & 14.0 \\
\hline cinco especies & & Croton collinus & 4 & 4 & 16 & 0.03 & 1.00 & 0.08 & 0.07 & 0.001 & 10.0 \\
\hline muestreadas, con & & Eriotheca ruizii & 14 & 3 & 56 & 0.09 & 0.75 & 0.06 & 14.74 & 0.114 & 26.0 \\
\hline ехсер & & Geoffroea striata & 1 & 1 & 4 & 0.01 & 0.25 & 0.02 & 0.26 & 0.002 & 3.0 \\
\hline olca; & & Loxopterygium huasan & 3 & 3 & 12 & 0.02 & 0.75 & 0.06 & 45.97 & 0.357 & 43.0 \\
\hline & & Schrebera americana & 1 & 1 & 4 & 0.01 & 0.25 & 0.02 & 1.39 & 0.010 & 4.0 \\
\hline & & Senna mollisima & 5 & 4 & 20 & 0.03 & 1.00 & 0.08 & 2.68 & 0.021 & 13.0 \\
\hline & & Tecoma stans & 20 & 4 & 80 & 0.12 & 1.00 & 0.08 & 1.42 & 0.011 & 21.0 \\
\hline & & Ximenia americana & 1 & 1 & 4 & 0.01 & 0.25 & 0.02 & 4.44 & 0.034 & 6.00 \\
\hline & & & 162 & & 648 & 1.00 & 12.75 & 1.00 & 128.86 & 1.000 & 300.0 \\
\hline
\end{tabular}
que en nuestro $\mathrm{N}^{\mathrm{o}}$ Indiv. , número de individuos por especie; $\mathrm{N}^{\circ} \mathrm{I} / \mathrm{T}$, número de veces que la especie se repite en los trabajo, con transectos; D, densidad; Dr, densidad relativa; F, frecuencia; Fr, frecuencia relativa; AB, área basal; excepción de E. ABr, área basal relativa; IVI, Índice de valor de importancia.

ruizii, cuyo IVI

resultó bastante

alto en el bosque Chaparrí así como para L. huasango, con valores similares, para C. lutea y B. graveolens, el IVI fue mayor en los BES La Menta y Timbes.

Por otro lado, resulta interesante observar que no obstante reconocerse que la presión humana sobre el bosque ha sido muy intensa en los últimos años, todavía es posible encontrar especies de alto valor comercial, con especímenes de gran porte como fue el caso de Loxopterygium huasango, Bursera graveolens, Erythrina smithiana y Ceiba trichistandra, lo que puede atribuirse a varios factores como la producción de grandes cantidades de semilla, escaso impacto antrópico, lugares inaccesibles, entre otros; al respecto, cabe indicarse que los árboles muestreados de mayor tamaño se encontraban en quebradas y

\begin{tabular}{|c|c|c|c|c|c|c|c|c|c|}
\hline Especie & $\begin{array}{c}\mathrm{N}^{\mathbf{0}} \\
\text { Indiv. }\end{array}$ & $\begin{array}{l}\mathbf{N}^{\mathbf{0}} \\
\mathbf{I} / \mathbf{T}\end{array}$ & D & Dr & $\mathbf{F}$ & Fr & $\begin{array}{c}\mathrm{AB} \\
\left(\mathbf{m}^{2}\right)\end{array}$ & $\overline{\mathrm{ABr}}$ & $\begin{array}{l}\text { IVI } \\
(\%)\end{array}$ \\
\hline $1 \begin{array}{l}\text { Bougainvillea } \\
\text { pachyphylla }\end{array}$ & 3 & 2 & 12 & 0.02 & 0.50 & 0.04 & 0.32 & 0.002 & 6.00 \\
\hline 2 Bursera graveolens & 10 & 4 & 40 & 0.05 & 1.00 & 0.08 & 14.96 & 0.08 & 20.0 \\
\hline 3 Capparis scabrida & 1 & 1 & 4 & 0.01 & 0.25 & 0.02 & 3.65 & 0.019 & 4.0 \\
\hline 4 Cassia sp. & 8 & 3 & 32 & 0.04 & 0.75 & 0.06 & 0.29 & 0.001 & 10.0 \\
\hline 5 Ceiba trichistandra & 7 & 1 & 28 & 0.04 & 0.25 & 0.02 & 38.88 & 0.198 & 25.0 \\
\hline 6 Cestrum auriculatum & 8 & 3 & 32 & 0.04 & 0.75 & 0.06 & 0.90 & 0.005 & 10.0 \\
\hline 7 Cissus sp. & 7 & 4 & 28 & 0.04 & 1.00 & 0.08 & 2.58 & 0.013 & 13.0 \\
\hline 8 Coccoloba ruiziana & 2 & 2 & 8 & 0.01 & 0.50 & 0.04 & 2.59 & 0.013 & 6.0 \\
\hline $\begin{array}{l}9 \text { Cochlospermum } \\
\text { vitifolium }\end{array}$ & 1 & 1 & 4 & 0.01 & 0.25 & 0.02 & 1.46 & 0.007 & 3.0 \\
\hline 10 Cordia lutea & 95 & 5 & 380 & 0.50 & 1.25 & 0.09 & 4.94 & 0.025 & 62.0 \\
\hline $\begin{array}{l}11 \text { Cordia } \\
\text { macrocephala }\end{array}$ & 3 & 2 & 12 & 0.02 & 0.50 & 0.04 & 0.22 & 0.001 & 6.0 \\
\hline 12 Croton collinus & 5 & 3 & 20 & 0.02 & 0.75 & 0.06 & 0.05 & 0.000 & 8.0 \\
\hline 13 Eriotheca ruizii & 3 & 2 & 12 & 0.0 & 0.5 & 0.04 & 33.28 & 0.169 & 22.0 \\
\hline 14 Erythrina smithiana & 15 & 4 & 60 & 0.08 & 1.00 & 0.08 & 47.16 & 0.240 & 40.0 \\
\hline 15 Leucaena trychodes & 1 & 1 & 4 & 0.01 & 0.25 & 0.02 & 22.83 & 0.016 & 14.0 \\
\hline $\begin{array}{l}16 \begin{array}{l}\text { Loxopterygium } \\
\text { huasango }\end{array}\end{array}$ & 2 & 2 & 8 & 0.01 & 0.50 & 0.04 & 14.36 & 0.073 & 12 \\
\hline 17 Myrcianthes sp. & 3 & 1 & 12 & 0.02 & 0.25 & 0.02 & 0.04 & 0.000 & 4.0 \\
\hline $\begin{array}{l}18 \text { Pisonia } \\
\text { macranthocarpa }\end{array}$ & 4 & 4 & 16 & 0.02 & 1.00 & 0.08 & 0.24 & 0.001 & 10.0 \\
\hline 19 Senna mollisima & 7 & 3 & 28 & 0.04 & 0.75 & 0.06 & 1.22 & 0.006 & 10.0 \\
\hline 20 Sida paniculata & 1 & 1 & 4 & 0.01 & 0.25 & 0.02 & 0.01 & 0.000 & 2.0 \\
\hline 21 Solanum amotapense & 1 & 1 & 4 & 0.01 & 0.25 & 0.02 & 0.06 & 0.000 & 2.0 \\
\hline 22 Tecoma stans & 1 & 1 & 4 & 0.01 & 0.25 & 0.02 & 2.06 & 0.010 & 4.0 \\
\hline 23 Ximenia americana & 2 & 2 & 8 & 0.01 & 0.50 & 0.04 & 4.54 & 0.023 & 7.0 \\
\hline & 190 & & 760 & 1.00 & 13.25 & 1.00 & 196.63 & 1.000 & 0.00 \\
\hline
\end{tabular}

$\mathrm{N}^{0}$ Indiv., número de individuos por especie; $\mathrm{N}^{\mathrm{o}} \mathrm{I} / \mathrm{T}$, número de veces que la especie se repite en los transectos; D, densidad; Dr, densidad relativa; F, frecuencia; Fr, frecuencia relativa; $\mathrm{AB}$, área basal; $\mathrm{ABr}$, área basal relativa; IVI, Índice de Valor de Importancia. 
peñascos de difícil acceso, además, que las comunidades campesinas vienen desarrollando ciertas actividades protección.

Índice de riqueza específica (IR), Índice de dominancia (ID), Índice de Diversidad (H') y Matrices de similaridad (IS).

Como se muestra en la tabla 7, el IR específica de Margalef, que expresa el número total de especies obtenido por un censo de la comunidad, indicó como mayor valor para el BES La Menta 3.55 en el transecto 2 y para el BES Timbes 3.77 en el transecto

4 , en tanto que para ambos BES fue mayor para Timbes con 4.19; en general el IR específica fue ligeramente similar en todos los transectos de cada bosque, variando entre 2.09 a 3.77 , con excepción del transecto 5 del bosque de Timbes con un valor 0.00 puesto que apenas registró una sola especie. Resulta conveniente precisar que en el cálculo de este índice, como cualquier otro que utilice logaritmos, el resultado dependerá del tipo de logaritmo que se emplee, es decir, si se utiliza logaritmo base $10 \mathrm{o}$ logaritmos neperianos (Moreno, 2001). El ID de Simpson, que expresa la probabilidad de que dos individuos tomados al azar de una muestra sean de la misma especie, indicó como mayor valor para La Menta 0.89 en el transecto 4 y para Timbes 0,85 en el transecto 3 , en tanto que para ambos bosques fue mayor para La Menta con 0.88; en general el ID fue, también, ligeramente similar en todos los transectos de cada bosque, variando entre 0.74 a 0.89 , siempre con excepción del transecto 5 de Timbes con un valor 0.00 puesto que apenas registró una sola especie. Los índices de dominancia de Shannon-Wiener $\left(\mathrm{H}^{\prime}\right)$ fueron mayores en los transectos 4 y 3, tanto en La Menta con 2.17 y 2.08 , respectivamente como en Timbes con 2.20 y 2.09 , respectivamente, lo que indicó que en estos transectos se presentó un mayor número de especies. El análisis global de cada BES mostró, a través del Índice de Shannon-Wiener, que el BES La Menta fue de mayor valor, con 2.36, respecto al BES Timbes, con 2.08, aún cuando tuvo un menor número de especies, denotando una distribución más equitativa de las especies.

En la tabla 8, las Matrices de Similaridad Bray y Curtis (\%) para el BES La Menta mostró que el transecto 1 tuvo mayor similaridad con el transecto 4 y éste a su vez con el transecto 3 y muy alta similaridad con el transecto 5; para el BES Timbes la mayor similaridad fue entre los transectos 3 con 4 y que el transecto 5 tuvo una débil similaridad con los demás transectos. Entre ambos BES la similaridad fue de $54.68 \%$

Tabla 8. Matrices de Similaridad Bray y Curtis (\%) para transectos de los BES La Menta y Timbes y la interacción de éstos.

\begin{tabular}{|c|c|c|c|c|c|}
\hline \multicolumn{6}{|c|}{ BES La Menta } \\
\hline & $\mathrm{T} 1$ & $\mathrm{~T} 2$ & T3 & $\mathrm{T} 4$ & T5 \\
\hline T1 & - & & & & \\
\hline $\mathrm{T} 2$ & 60.28 & & & & \\
\hline $\mathrm{T} 3$ & 57.73 & 59.55 & & & \\
\hline $\mathrm{T} 4$ & 67.17 & 56.49 & 68.64 & & \\
\hline $\mathrm{T} 5$ & 62.01 & 50.05 & 62.13 & 83.81 & - \\
\hline BES T & bes & & & & \\
\hline & $\mathrm{T} 1$ & $\mathrm{~T} 2$ & $\mathrm{~T} 3$ & $\mathrm{~T} 4$ & $\mathrm{~T} 5$ \\
\hline T1 & - & & & & \\
\hline $\mathrm{T} 2$ & 55.05 & & & & \\
\hline T3 & 53.32 & 58.47 & & & \\
\hline T4 & 54.93 & 64.54 & 74.03 & & \\
\hline T5 & 15.05 & 22.45 & 17.91 & 16.58 & - \\
\hline BES I & Menta - & nbes & & & \\
\hline & $\mathrm{T} 1$ & $\mathrm{~T} 2$ & & & \\
\hline $\mathrm{T} 1$ & - & & & & \\
\hline $\mathrm{T} 2$ & 54.68 & - & & & \\
\hline
\end{tabular}

Aspectos ecológicos y conservación de las especies.

Las características geomorfológicas, climáticas (temperatura y pluviosidad) e hidrográficas de la cuenca del río Chipillico, así como la similaridad en los análisis de suelo y la poca variación en la latitud, longitud y altitud, entre los BES La Menta y Timbes, determinaron la ocurrencia de características edáficas y composición florística bastante parecidas. A ello 
habría de sumar haber estado expuestos a la misma presión humana de deforestación $\mathrm{y}$ pastoreo de caprinos y vacunos, razón por la cual la especie dominante sea $C$. lutea, un arbusto de rápido y profuso crecimiento, llegando incluso en el transecto 5 de Timbes a constituirse en la única especie muestreada. Otras especies de reconocida importancia forestal como Loxopterygium huasango, Caesalpinia glabrata, Bursera graveolens, Eriotheca ruizii, Schrebera americana, Ximenia americana, Cochlospermum vitifolium y Geoffroea striata, aparecieron con muy pocos individuos e incluso algunas especies con apenas un único individuo; sin embargo, eran árboles adultos, lo que de alguna manera hacía suponer que estarían siendo protegidos por las comunidades campesinas. Otras especies arbóreas como Pithecellobium multiflorum (Angolo), Muntingia calabura (Cerezo), Capparis mollis (Suno) y Celtis iguanaea (Palo blanco), fueron observadas en los alrededores de las áreas de muestreo, en tanto que Tabebuia billbergii (Guayacán), Terminalia valverdae (Guarapo) y Cedrela sp. (Cedro), no fueron observadas en ningún caso.

La composición florística de los BES La Menta y Timbes está en relación con uno de los seis grupos de vegetación establecidos por La Torre-Cuadros \& Linares-Palomino (2008), estudiando la vegetación estacional de las llanuras costeras de Piura; estos autores determinaron que entre los 390 y $750 \mathrm{~m}$ se encuentran especies como Eriotheca ruizii, Pithecellobium excelsum y Erythrina smithiana y otras como Maytenus sp.1, Celtis iguanaea, Leucaena trichodes, Albizia multiflora, Acacia macracantha, Loxopterygium huasango y Bursera graveolens, la mayoría reportadas en nuestro estudio. Esta vegetación fue reconocida por Weberbauer (1922, 1930) como "montes macrotérmicos, compuestos de árboles, arbustos y cactáceas, donde, terminada la estación lluviosa, la mayor parte de los árboles y arbustos se deshojan".

Atendiendo la clasificación de La Torre-Cuadros \& Linares-Palomino (2008), las diferencias marcadas entre el número de especies e individuos presentes en cada estrato altitudinal del BES están condicionadas por la demanda del recurso edáfico, hidrológico y de humedad, por lo tanto, el desarrollo de las especies y la distribución restringida de los individuos en estos bosques, se limita a la estacionalidad climática de la región que condiciona la dinámica del bosque (LealPinedo \& Linares-Palomino, 2005), lo que coincide con observaciones realizadas por Gentry \& Ortiz (1993) basados en datos de bosques húmedos $\mathrm{y}$ lluviosos; sin embargo, resulta significativo la ausencia total en los BES La Menta y Timbes de Prosopis pallida (Humb. \& Bonpl. ex Willd.) Kunth (algarrobo) y Capparis scabrida Kunth (zapote), dos especies emblemáticas del bosque seco, lo que ciertamente no obedece a factores estrictamente edáficos e hídricos sino también a la acción depredadora del hombre y del ganado caprino, en especial en este último caso sobre la regeneración natural. En todo caso, fenoménos naturales como el último evento El Niño de los años 1997-1998 alteran significativamente la dinámica poblacional del bosque estacionalmente seco, tal como fue reportado con la vegetación de los algarrobales, con el algarrobo (Prosopis pallida) como especie dominante (Gushiken et al., 2001).

En general, en el BES La Menta fueron registrados 162 árboles y arbustos en el muestreo (648/ha) y en el BES Timbes 190 árboles y arbustos en el muestreo (760/ha). En el bosque seco Piedra del Toro fueron registrados 64 arb./Ha en el bosque seco ralo, destacando Eriotheca ruizii, Loxopterygium huasango y Prosopis pallida, $153.1 \mathrm{arb} . / \mathrm{Ha}$ en el bosque seco semidenso, destacando Bursera graveolens, Eriotheca ruizii y Erythrina smithiana y 275.8 arb./Ha en el bosque seco denso, destacando Bursera graveolens, Bougainvillea pachyphylla y Loxopterygium huasango (PEBANINSA, 2003). En el bosque seco La Menta fueron registrados 138.2 arb./ha en el bosque seco semidenso, destacando Eriotheca ruizii, Bursera graveolens y Loxopterygium huasango y 159.9 arb./ha en el bosque seco denso, destacando Eriotheca ruizii y Bursera graveolens (HPI-PERÚ, 2007). Estos datos nos permiten inferir que el proceso de deterioro del bosque seco ecuatorial continúa a un ritmo sostenido, resultando necesario implementar urgentemente programas de conservación y reforestación, tal como ya ha sido iniciado por la Asociación Chira.

\section{Conclusiones.}

1. Las características edáficas permitió clasificar a los suelos de los BES La Menta y Timbes en las categorías Orden Entisoles y suborden Orthents, propias de áreas geográficas con clima y vegetación de bosque estacionalmente seco.

2. La composición florística, entre árboles y arbustos, con un DAP $\geq 1.0 \mathrm{~cm}$, para el BES La Menta fue de 162 individuos en el área muestreada (648/ha) que correspondió a 18 especies, 16 géneros y 15 familias y para el BES Timbes fue de 190 individuos (760/ha) que correspondió a 23 especies, 22 géneros y 19 familias.

3. El área basal, por el área evaluada, fue de $128.86 \mathrm{~m}^{2}$ para La Menta, destacando Loxopterygium huasango con $45.97 \mathrm{~m}^{2}$ y $196.63 \mathrm{~m}^{2}$ para Timbes, destacando Erythrina smithiana con $47.13 \mathrm{~m}^{2}$. El Índice de Valor de Importancia fue $50.0 \%$ para Bursera graveolens en La Menta y $62.0 \%$ para Cordia lutea en Timbes.

4. El escaso número de especímenes de gran porte y óptima vocación forestal, que aún persisten, indicaría que estarían siendo protegidos en parte por su difícil acceso y en parte por el cuidado de los 
pobladores rurales, lo cual alentaría el establecimiento de programas de reforestación.

\section{Agradecimientos.}

A la Xunta de Galicia (España) y al Proyecto Binacional Catamayo - Chira, por el financiamiento de la investigación, a Jorge Chanamé Céspedes, profesor de la Universidad Nacional Pedro Ruiz Gallo de Lambayeque, por su contribución en el análisis estadístico y a Alexander Huamán Mera y Jeanine Córdova Risco, por el apoyo técnico en la preparación del manuscrito.

\section{Literatura citada.}

Aguilar P. 1994. Características faunísticas del norte del Perú. Arnaldoa 2: 77-102.

Aguirre Z. \& Delgado T. 2005. Vegetación de los bosques secos de Cerro Negro-Cazaderos, Occidente de la Provincia de Loja. En: M.A. Vásquez, J.F. Freira y L. Suárez (editores) Biodiversidad en los bosques secos de la zona de Cerro Negro-Cazaderos, occidente de la provincia de Loja: un reporte de las evaluaciones ecológicas y socioeconómicas rápidas. EcoCiencia, MAE y Proyecto Bosque Seco, Quito.

Aguirre Z. \& Kvist L.P. 2005. Composición florística y estado de conservación de los bosques secos del suroccidente del Ecuador. Lyonia 8:41-67.

Aguirre Z., Cueva E. \& Merino B. 2001. Evaluación ecológica rápida de la vegetación en los bosques secos de La Ceiba y Cordillera Arañitas, provincia de Loja, Ecuador. En: M.A. Vásquez, M. Larrea y L. Suárez et al. (editores). Biodiversidad en los bosques secos del suroccidente de la provincia de Loja. EcoCiencia, Ministerio del Ambiente, Herbario LOJA y Proyecto Bosque Seco, Quito.

Aguirre-Mendoza J., Linares-Palomino R. y Kvist L.P. 2006. Especies leñosas y formaciones vegetales en los bosques estacionalmente secos de Ecuador y Perú. Arnaldoa 13: 324-350.

Alfaro E.A. Alvarado A. \& Chaverri A. 2001. Cambios edáficos asociados a tres etapas sucesionales de bosque tropical seco en Guanacaste, Costa Rica. Agronomia Costarricense 25: 7-20.

APG (Angiosperm Phylogeny Group). 1998. An ordinal classification for the families of flowering plants. Annals of the Missouri Botanical Garden 85:531-553.

APG (Angiosperm Phylogeny Group). 2003. An update of the Angiosperm Phylogeny Group classification for the orders and families of flowering plants: APG II. Botanical Journal of the Linnean Society 141:399-436.

Armijos D. \& Valarezo K. 2010. Diversidad de anfibios y reptiles de un bosque seco en el sur occidental del Ecuador. Ecología Forestal (Loja) 1: 30-36.

Bernex de Falen N. \& Revesz B. 1988. Atlas Regional de Piura. CIPCA-UPCP, Piura. 208 pp.

Brack A. \& Mendiola C. 2004. Ecología del Perú. PNUD. Asociación Editorial Bruño. Lima, Perú. 495 pp.

Bray J.R. \& Curtis J.T. 1957. An ordination of the upland forest communities of southern Wisconsin. Ecological Monographs. 27: 325-349.

Bridgewater S., Pennington R.T., Reynel C.A., Daza A. y Pennington T.D. 2003. A preliminary floristic and phytogeographic analysis of the woody flora of seasonally dry forests in northern Peru. Candollea 58: 129-148.

CDC, Centro de Datos para la Conservación. 1992. Estado de conservación de la diversidad natural de la región noroeste del Perú. Universidad Nacional Agraria La Molina, Lima, Perú.

Cerón C.E., Reyes C.I. \& Vela C. 2006. Características botánicas de la Reserva Militar y Ecológica Arenillas, El Oro - Ecuador. Cinchonia 7: 115-130.

Certini G. 2005. Effects of fire on properties of forest soils: a review. Oecologia 143: 1-10.

Chazdon R.L. 2003. Tropical forest recovery: legacies of human impact and natural disturbances. Perspect. Plant Ecology 6: 51-71.

Clark D.B, Palmer M.W. \& Clark D.A. 1999. Edaphic factors and the landscape-scale distributions of tropical rain forest trees. Ecology 80: 2662-2675.

Cuevas, E. 1995. Biology of the belowground system of tropical dry forest. Páginas 362-383. En: S.H Bullock, H.A. Mooney \& E. Medina (editores). Seasonally dry tropical forests. Cambridge University, Cambridge, Reino Unido.

García-Villacorta R. 2009. Diversidad, composición y estructura de un hábitat altamente amenazado: los bosques estacionalmente secos de Tarapoto, Perú. Revista Peruana de Biología 16: 81-92.

Gentry A.H. 1982. Patterns of neotropical diversity. Evolutionary Biology 15: 1-84.

Gentry, A.H. 1995. Diversity and floristic composition of neotropical dry forests. Páginas 146-194. En: S.H Bullock, H.A. Mooney \& E. Medina (editores). Seasonally dry tropical forests. Cambridge University, Cambridge, Reino Unido.

Gentry A.H. \& Ortiz R. 1993. Patrones de Composición Florística en la Amazonía Peruana. En: R. Kalliola, M. Puhakka \& W. Danjoy (editores). Amazonía Peruana Vegetación Húmeda en el Llano Subandino. Proyecto Amazonía de la Universidad de Turku, Finlandia y ONERN-Perú, Jyvaskyla.

Goldberg D.E. 1982. The distribution of evergreen and deciduous trees relative to soil type: an example from the Sierra Madre, México, and a general model. Ecology 63: 942-951

Gushiken S., Acuña, T. \& Torres, J. 2001. Dinámica poblacional de los algarrobales (Prosopis pallida) y El Niño en la costa norte del Perú. Páginas 213-223. En: J. Tarazona, W. Arntz y E. Castillo (eds.). El Niño en América Latina, impactos biológicos y sociales. Consejo Nacional de Ciencia y Tecnología, Lima, Perú.

Holdridge L. 1982. Ecología basada en zonas de vida. IICA, San José, Coata Rica. 216 pp.

HPI-PERÚ (Heifer Project International). 2007. Inventario del potencial del bosque seco de la Comunidad Campesina La Menta, Piura (Perú). Informe Interno. 62 pp.

INRENA-DGEPRN. 1998. Mapa de bosques secos del departamento de Piura. Lima. 90 pp.

Jackson M.L. 1982. Análisis químico de suelos. Cuarta Edición. Ed. Omega, S.A., Barcelona. 662 pp.

Josse C. \& Balslev H. 1994. The composition and structure of a dry, semideciduous forest in western Ecuador. Nordic Journal of Botany 14: 425-434.

La Torre-Cuadros M.A. \& Linares-Palomino R. 2008. Mapas y clasificación de vegetación en ecosistemas 
Agosto - Diciembre 2011

estacionales: un análisis cuantitativo de los bosques secos de Piura. Revista Peruana de Biología. 15: 31-42.

Leiva J.A., Mata R., Rocha O.J. \& Gutiérrez M.V. 2009. Cronología de la regeneración del bosque tropical seco en Santa Rosa, Guanacaste, Costa Rica. I. Características edáficas. Revista Biología Tropical 57: 801-815.

Leal-Pinedo J.M. \& Linares-Palomino R. 2005. Los bosques secos de la Reserva de Biosfera del Noroeste (Perú): Diversidad arbórea y estado de conservación. Caldasia 27: 195-211.

Linares-Palomino R. 2005. Spatial distribution patterns of trees in a seasonally dry forest in the Cerros de Amotape National Park, northwestern Peru. En: Bosques relictos del NO de Perú y SO del Ecuador. Revista Peruana de Biología 12: 317-326.

Linares-Palomino R. \& Pennington R.T. 2007. Lista anotada de plantas leñosas en bosques estacionalmente secos del Perú - una nueva herramienta en internet para estudios taxonómicos, ecológicos y de biodiversidad. Arnaldoa 14: $149-152$

Linares-Palomino R. \& Ponce-Álvarez S. 2009. Structural patterns and floristics of a seasonally dry forest in Reserva Ecológica Chaparri, Lambayeque, Peru. Tropical Ecology 50(2): 305-314.

Linares-Palomino R., Kvist L.P., Aguirre-Mendoza Z. \& Gonzales-Inca C. 2010. Diversity and endemism of woody plant species in the Equatorial Pacific seasonally dry forests. Biodiversity and Conservation 19: 169-185.

López J. \& López, J. 1990. El diagnóstico de suelos y plantas: Métodos de campo y laboratorio. Tercera Edición. Ed. Mundi-Prensa, España. 363 pp.

Magurran A.E. 1988. Ecological diversity and its measurement. Princeton University Press, New Jersey, $179 \mathrm{pp}$.

Marcelo-Peña J.L. 2008. Vegetación leñosa, endemismos y estado de conservación en los bosques estacionalmente secos de Jaén, Perú. Revista Peruana de Biología 15: 4352.

Martos J.R., Scarpati M., Rojas C. \& Delgado G.E. 2009. Fenología de algunas especies que son alimento para la pava aliblanca Penelope albipennis. Revista Peruana de Biología 15: 51-58.

Mittermeier R.A., Goettsch C. \& Robles Gil P. 1997. Megadiversidad: Los países biológicamente más ricos del mundo. CEMEX, SA. de CV. México.
Mittermeier R.A., Robles Gil P., Hoffman M., Pilgrim J., Brooks T., Goettsch Mittermeier C., Lamoreux J. \& Da Fonseca G.A.B. 2005. Hotspots revisited: Earth's biologically richest and most threatened terrestrial ecoregions. Conservation International. Washington.

Moreno C.E. 2001. Métodos para medir la biodiversidad. M\&T - Manuales y Tesis SEA, vol. 1. CYTED, ORCYT/UNESCO \& SEA. Zaragoza, España.

ONERN, Oficina Nacional de Evaluación de Recursos Naturales. 1976. Mapa ecológico del Perú. Guía explicativa. Lima, Perú. 147 pp.

PEBANINSA, PAEN/GTZ. 2003. Evaluación del potencial forestal del bosque seco Piedra del Toro. PEBANINSA, PAEN/GTZ, Plan Piura. 105 pp.

Proyecto Algarrobo. 1993. Mapa e inventario forestal de los bosques secos de Lambayeque. Memoria explicativa. CEIMAD-Proyecto Algarrobo, Chiclayo, Perú.

Russell A.E., Raich J.W., Valverde-Barrantes O.J. \& Fisher R.F. 2007. Tree species effects on soil properties in experimental plantations in tropical moist forest. Soil Science Society of the American Journal 71: 1389-1397.

Sagástegui A., Dillon M.O, Sánchez I., Leiva S. \& Lezama P. 1999. Diversidad Florística del Norte del Perú. Tomo I. Trujillo; Edit. Graficart.

Soil Survey Staff, 2010. Keys to Soil Taxonomy. $11^{\text {th }}$ ed. USDA-Natural Resources Conservation Service, Washington, DC.

Weberbauer A. 1922. Die Vegetationskarte der peruanischen Anden zwischen $5^{\circ}$ und $17^{\circ} \mathrm{S}$. Petermanns Geographische Mitteilungen 68: 89-91.

Weberbauer A. 1930. Die Pflanzendecke Nordperus im Departamento Tumbez una angrenzenden Teilen des Departamento Piura $\left(3^{\circ} 30^{\circ}-5^{\circ} \mathrm{s}\right.$. Br. $)$. Botanische Jahrbücher 63: 29-48.

Wilkie P., Argent G., Cambell E. \& Saridan A. 2004. The diversity of 15 ha of lowland mixed dipterocarp forest. Central Kalimantan. Biodiversity and Conservation 13: 695-708.

WRI. World Resources Institute. 1997. World Resources 1996-97: The urban environment. Washington, USA.

Zevallos P. 1986. Caracterización dendrológica de 30 especies forestales de Lambayeque. Tesis Universidad Nacional Agraria La Molina. Lima - Perú. 142 p.

\footnotetext{
${ }^{1}$ Jardín Botánico de Valencia. Universitat de València, Valencia, ESPAÑA. maria.rasal@cerai.org

${ }^{2}$ Asociación Chira - Centro de Formación Binacional, Mallares, Sullana, Piura, PERÚ. jtroncos@asociacionchira.org

3 Asociación Chira - Centro de Formación Binacional, Mallares, Sullana, Piura, PERÚ. carlidur@hotmail.com

${ }^{4}$ Asociación Chira - Centro de Formación Binacional, Mallares, Sullana, Piura, PERÚ. pgranda@hotmail.com

${ }^{5}$ Asociación Chira - Centro de Formación Binacional, Mallares, Sullana, Piura, PERÚ. dquevedo2009@hotmail.com

${ }^{6}$ Facultad de Ciencias Biológicas, Universidad Nacional Pedro Ruiz Gallo, Lambayeque, PERÚ. crojasi2002@yahoo.es

${ }^{7}$ Facultad de Ciencias Biológicas, Universidad Nacional Pedro Ruiz Gallo, Lambayeque, PERÚ. guidelg2001@yahoo.es
} 